\author{
Matthias Haenggi \\ Sina Blum \\ Ruth Brechbuehl \\ Anna Brunello \\ Stephan M. Jakob \\ Jukka Takala
}

\section{Effect of sedation level on the prevalence of delirium when assessed with CAM-ICU and ICDSC}

Received: 15 November 2012

Accepted: 12 July 2013

Published online: 7 August 2013

(C) Springer-Verlag Berlin Heidelberg and ESICM 2013

S. Blum and R. Brechbuehl contributed equally.

Trial registration: German Clinical Trials Register DRKS00003437

(http://www.drks.de)

Electronic supplementary material

The online version of this article (doi:10.1007/s00134-013-3034-5) contains supplementary material, which is available to authorized users.

M. Haenggi (四) S. Blum •

R. Brechbuehl - A. Brunello ·

S. M. Jakob · J. Takala

Department of Intensive Care Medicine,

Bern University Hospital (Inselspital),

University of Bern, Freiburgstrasse,

3010 Bern, Switzerland

e-mail: matthias.haenggi@insel.ch

Tel.: +41-31-6323029

Fax: +41-31-6321771
Abstract Purpose: We hypothesized that reduced arousability (Richmond Agitation Sedation Scale, RASS, scores -2 to -3 ) for any reason during delirium assessment increases the apparent prevalence of delirium in intensive care patients. To test this hypothesis, we assessed delirium using the Confusion Assessment Method for the Intensive Care Unit (CAM-ICU) and Intensive Care Delirium Screening Checklist (ICDSC) in intensive care patients during sedation stops, and related the findings to the level of sedation, as assessed with RASS score.

Methods: We assessed delirium in 80 patients with ICU stay longer than $48 \mathrm{~h}$ using CAM-ICU and ICDSC during daily sedation stops. Sedation was assessed using RASS. The effect of including patients with a RASS of -2 and -3 during sedation stop ("light to moderate sedation", eye contact less than $10 \mathrm{~s}$ or not at all, respectively) on prevalence of delirium was analyzed. Results: A total of 467 patient days were assessed.
The proportion of CAM-ICU-positive evaluations decreased from 53 to $31 \%(p<0.001)$ if assessments from patients at RASS $-2 /-3$ (22\% of all assessments) were excluded. Similarly, the number of positive ICDSC results decreased from 51 to $29 \%$ $(p<0.001)$. Conclusions: Sedation per se can result in positive items of both CAM-ICU and ICDSC, and therefore in a diagnosis of delirium. Consequently, apparent prevalence of delirium is dependent on how a depressed level of consciousness after sedation stop is interpreted (delirium vs persisting sedation). We suggest that any reports on delirium using these assessment tools should be stratified for a sedation score during the assessment.

\section{Keywords CAM-ICU (Confusion} Assessment Method for the Intensive Care Unit) · ICDSC (Intensive Care Delirium Screening Checklist) . Intensive care medicine - Delirium . Sedation

\section{Introduction}

Agitation and hallucinations but also features of hypoactive delirium are common in intensive care patients [1]. Delirium has been associated with prolonged intensive care unit and hospital stay, long-term impairment of cognitive function, and increased mortality [2-5]. Tools to diagnose delirium in intensive care patients have been developed; these include the Confusion Assessment Method for the Intensive Care Unit (CAM-ICU) [6, 7] and the Intensive Care Delirium Screening Checklist (ICDSC) [8]. The use of these tools has led to highly variable occurrence rates for delirium-ranging from 16 to $87 \%$-and high prevalence of hypoactive delirium subtype being reported [5, 6, 8-12].

The diagnosis of delirium is particularly problematic in intubated patients because of problems of communication 
and the common use of sedative and analgesic drugs. Both CAM-ICU and ICDSC have been validated after excluding comatose patients but including patients with variable degrees of alertness, ranging from brief eye opening to vocal or to fully awake or severely agitated $[6-9,13]$.

Most commonly used sedative and analgesic agents have prolonged effects even if temporarily stopped, especially after prolonged exposure and in patients with multiple organ dysfunction, so persisting effects of sedative and analgesic drugs are likely to confound the assessment of delirium. This concept is supported by the predominance of hypoactive delirium in the published studies [5, 6, 8-11]. Distinguishing between the remaining effects of sedation and delirium is relevant especially when testing possible therapeutic pharmacologic interventions or preventive measures for delirium.

We hypothesized that reduced arousability (Richmond Agitation Sedation Scale, RASS, scores -2 to -3 ) for any reason during delirium assessment increases the apparent prevalence of delirium in intensive care patients. To test this hypothesis, we assessed delirium using CAMICU and ICDSC in intensive care patients during sedation stops, and related the findings to the level of sedation, as assessed with RASS score.

\section{Materials and methods}

The study was performed in a 30-bed, tertiary care, closed multidisciplinary intensive care unit. The study protocol was reviewed and approved by the Medical Research Ethics Committee of the Canton of Bern and informed consent was obtained from a close relative, with deferred consent from the patient once his/her condition had improved.

Between 2 February 2008 and 28 April 2008, all patients who were mechanically ventilated and sedated for more than $48 \mathrm{~h}$, aged at least 18 years, and fluent in German or French were screened. We excluded patients with pre-existing conditions interfering with assessment of CAM-ICU (e.g., tetraplegia, deafness, blindness, and overt dementia), and those who declined deferred consent. All patients were sedated using an institutional sedation protocol including sedation goals and daily sedation stops [14], details of which are provided in the electronic supplementary material (ESM). Delirium assessment with CAM-ICU and ICDSC is described in detail in the ESM. The study patients were assessed by the clinical team once daily during the sedation stop. The research team supervised the clinical team in the beginning, and provided help on request afterwards. In the rare case of disagreement of delirium scoring within the clinical team or between clinicians and the research team, the arguments were discussed and an agreement found. No independent assessments were done by the research team, as reliability and validity of the two scores have been reported to be high, and both instruments are claimed to be easy to use in clinical practice $[15,16]$. Daily evaluation was discontinued when the patient was free of sedation and did not present any neurological deficitsincluding delirium-for three consecutive days, or was discharged from the ICU.

Basic demographic data, reason for ICU admission, and SAPS II (Simplified Acute Physiology Score, with the Glasgow Coma Scale set as normal unless known otherwise) were recorded initially, followed by daily recording of mechanical ventilation, use of sedative drugs, RASS, CAM-ICU, and ICDSC.

CAM-ICU and ICDSC were assessed as proposed by their developers. For the purpose of the study, patients were classified as "comatose" if RASS remained at level -5 or -4 throughout the sedation stop, regardless of cause. Some sedation stops had to be discontinued for medical reasons, mainly because of agitation with threat of self-harm, or cardiovascular or respiratory instability. If RASS was positive at that time and the patient was not participating in assessment or comprehending instructions, inattention was considered to be present, and these events were categorized as CAM-ICU and ICDSC positive. When assessments were not done, we recorded the reason.

Low RASS scores after a sedation stop may be due to persisting sedation, encephalopathy, or their combination. In order to evaluate the relevance and magnitude of this problem to delirium assessment, patients with RASS -2 to -3 (light to moderate sedation, eye contact is maintained for less than $10 \mathrm{~s}$ at RASS -2 or not at all at RASS -3) were handled in three different ways in assessing delirium:

1. Included as proposed by the original publications of CAM-ICU and ICDSC (i.e., all patients included).

2. Defined as "not assessable" because of presumed ongoing sedation despite sedation stop (i.e., all patients at RASS -2 to -3 excluded).

3. Considering "not assessable" only those events, where according to the investigator, the patient was unable to maintain vigilance to understand the instructions of the letters attention test or the alternating pictures test of CAM-ICU. According to the original definition of CAM-ICU and ICDSC, these patients should be considered to have the features "acute onset of mental status changes or a fluctuating course", "inattention", "disorganized thinking", and "altered level of consciousness" positive (CAM-ICU), and at least the features "altered level of consciousness", "inattention", and "psychomotor agitation or retardation" of ICDSC positive.

Data are presented as mean \pm standard deviation, or frequencies. Because not all patients had the same number 
of assessments, the probability of a patient being classified as delirious was calculated by modeling the response using a mixed effects logistic regression. Test day was nested within patient ID, and both were included as random effects in the models. The identification method (original/ RASS -2 to -3 excluded/subjective) was included as a categorical factor. The probabilities of having died by day 28 and by day 90 if having been identified as delirious at least once or never were analyzed separately by each method of assessment. Logistic regressions were used with death considered an event, and being identified as delirious at least once or never coded as a binary explanatory variable. Comparisons of length of stay in the ICU and hospital of patients if identified as delirious at least once or never were performed with a one-way analysis of variance on ranks (Kruskal-Wallis). Further details of statistical analyses are indicated in the ESM. All tests were considered significant at a level of $p<0.05$. Analyses were performed using either Stata version 12.1 (Stata Statistical Software: Release 12. College Station, TX: StataCorp LP) (mixed model and regression analysis, done by the Clinical Trial Unit, University of Bern, Bern, Switzerland) or SigmaStat 3.5 (Systat Software GmbH, Erkrath, Germany) (all other analysis).

\section{Results}

A total of 87 consecutive patients were screened during three consecutive months and 80 patients were included (Table 1). Seven patients or their relatives refused deferred consent/declined participation, including one patient who was transferred to another hospital before consent could be obtained.

There were 467 scheduled sedation stops $(5.9 \pm 5.0$ evaluations per patient); on 333 study days sedation was used (4.2 \pm 4.2 days per patient). A total of 54 sedation stops $(16 \%)$ were not performed owing to patient instability, patient was not available in the operating room or radiology, and two additional sedation stops $(<1 \%)$ were not done because poor tube tolerance and pain despite of sedation/analgesia prohibited the pause of sedation and analgesia. In 65 instances (14\%) the delirium assessment was not performed owing to patient instability or absence while undergoing surgery or diagnostics $(n=54,11 \%)$, staff time constraints $(n=2,<1 \%)$, or for undocumented reasons $(n=9,2 \%)$. A total of 402 screenings for delirium assessments were done. In 78 (19\%) instances the patients were comatose with RASS -4 to -5 . In the $48 \mathrm{~h}$ before study entry and at day 1 continuous sedation was predominant; thereafter the use of continuous, intermittent, or no sedation at all was well balanced (Table 1 in ESM), and continuous sedation was predominantly done with propofol (Table 1 in ESM).
Table 1 Basic demographic and admission data

\begin{tabular}{ll}
\hline & Total $(n=80)$ \\
\hline Age (mean \pm SD) & $61 \pm 17$ \\
Men, $n(\%)$ & $53(66)$ \\
SAPS II score (mean $\pm \mathrm{SD})$ & $55 \pm 18$ \\
ICU admission, $n(\%)$ & \\
Medical & $37(46)$ \\
Surgical & $25(31)$ \\
Trauma & $18(23)$ \\
Type of sedation in the 48 h before study entry, $n(\%)$ \\
Continuous & $65(82)$ \\
Intermittent & $13(16)$ \\
Both & $1(1)$ \\
Data missing & $1(1)$ \\
\hline
\end{tabular}

10 patients $(13 \%)$ had pre-existing neurological disorders, 32 patients $(40 \%)$ were admitted for neurological disorders or neurotrauma. Details of admission diagnosis, pre-existing neurological disorders, and neuro/neuro-trauma admissions are provided in Table 6 in the ESM

CAM-ICU was positive in a total of $53 \%$ of assessments, negative in $27 \%$, and not assessable in $19 \%$ owing to coma (=RASS -4 or -5 ). In $23 \%$ of assessments, patients remained moderately sedated (RASS -2 to -3$)$ despite the sedation stop. In $58 \%$ of these assessments at RASS -2 to -3 , the investigators considered the patient insufficiently vigilant to follow instructions (Fig. 1).

Details on delirium assessments are indicated in the ESM. Ninety-two of the 215 CAM-ICU positive findings (42\%) were obtained during RASS -2 to -3 and all but two of the 92 assessment made at RASS -2 to -3 were CAM-ICU positive. If all the assessments at RASS -2 to -3 were considered as not assessable, $31 \%$ of all assessments were CAM-ICU positive, $27 \%$ CAM-ICU negative, and $42 \%$ not assessable owing to either coma or possible persisting sedation. If only those RASS -2 to -3 assessments, where the investigator considered the patient insufficiently vigilant to follow the instructions, were considered not assessable, $40 \%$ were CAM-ICU positive, $27 \%$ CAM-ICU negative, and $33 \%$ not assessable owing to either coma or possible persisting sedation (Fig. 1). The probability of being classified as delirious in the mixed model was $45 \%$ if all CAM-ICU positive assessments were included, $20 \%$ if all positive assessments at RASS -2 to -3 were excluded, and $29 \%$ if only those assessments where the investigator deemed the patient insufficiently vigilant to follow the instructions were excluded $(p<0.001)$. The corresponding numbers when ICDSC was used are 42, 20, and $28 \%$ (Fig. 2, $p<0.001$ in the mixed model).

Regarding the incidence, $71 \%$ of all patients were CAM-ICU positive at least once, $18 \%$ were CAM-ICU negative, and $11 \%$ remained comatose (RASS -4 or -5 ) throughout the study. A total of $19 \%(n=11)$ of CAM-ICU positive patients were positive only during 
Fig. 1 Flowchart of all CAM-ICU assessments
CAM-ICU

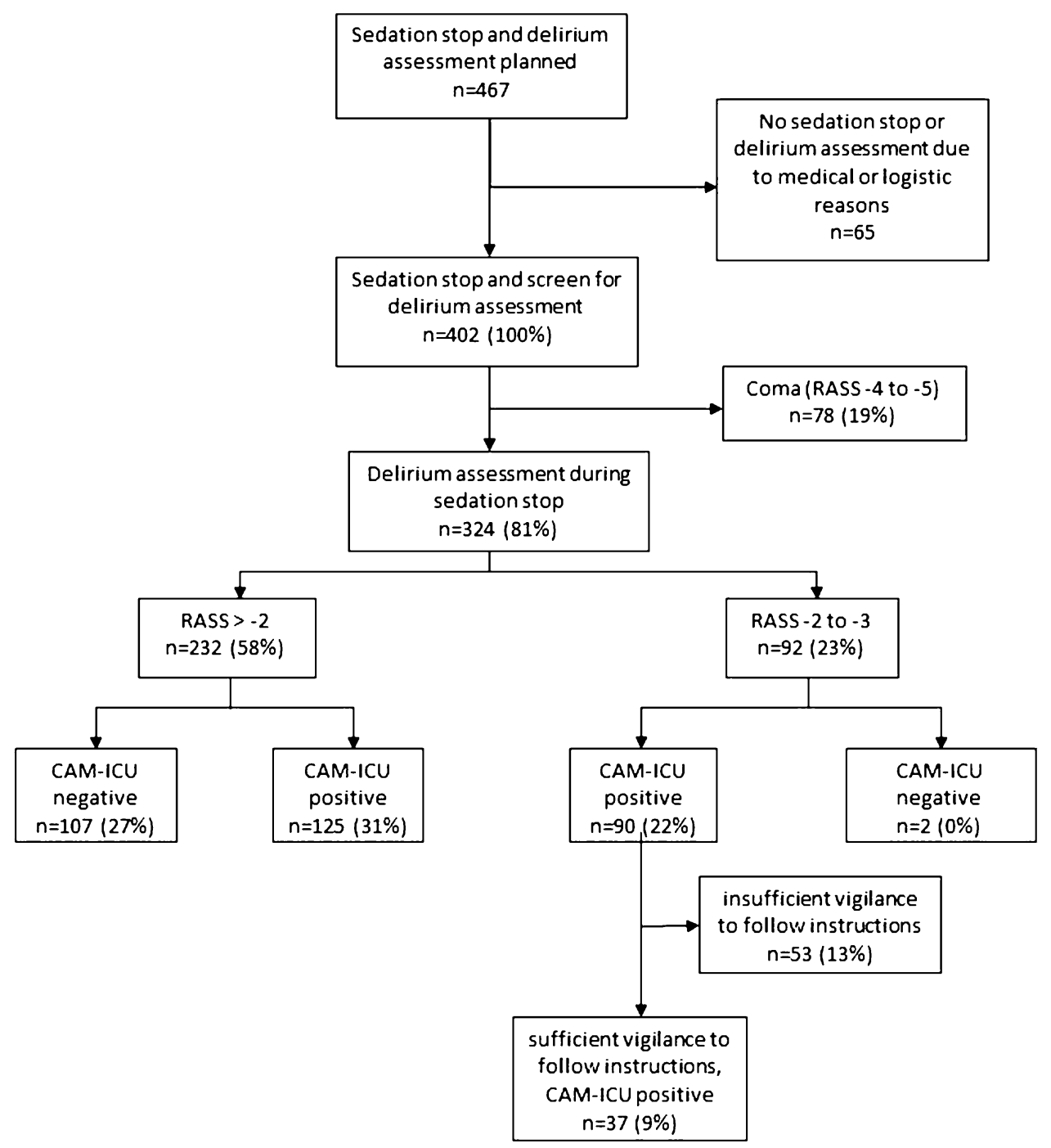

assessments with RASS -2 to -3 . Accordingly, if assessments at RASS -2 to -3 were considered not assessable, $58 \%$ of patients were CAM-ICU positive, $25 \%$ CAM-ICU negative, and $17 \%$ not assessable owing to either coma or persisting sedation $(p=0.099)$. The corresponding numbers in the third group are 66,18 , and $16 \%(p=0.609$; Fig. 3).

Delirium incidence and effects of level of sedation were similar when patients were assessed with ICDSC (Fig. 4).

When patients with neurological admission diagnoses $(n=32)$ were excluded from the analysis, the results were essentially similar (ESM Table 2).

The 11 patients who were evaluated CAM-ICU positive only at RASS -2 to -3 are similar to the total population. Details of demographics, SAPS, admission diagnosis, and sedation regimes are listed in Table 3 in the ESM.

Delirium presented predominantly in the hypoactive form; details to the distribution are shown in Table 4 in the ESM.

The only significant differences in ICU or hospital length of stay (LOS) between patients with or without delirium were the ICU-LOS if assessed with ICDSC, regardless of how delirium was classified. The study was not designed to find explanatory variables for that. The 28-day and 90-day mortalities tended to be higher in patients with delirium, but none of the differences was statistically significant (Table 5 in ESM). Patients with persisting coma (RASS -4 to -5 in all assessments) had higher mortality than those without coma (67 vs $20 \%$ at 28 days, $p=0.006$; 67 vs $25 \%$ at 90 days, $p=0.019$ ). 
Fig. 2 Flowchart of all ICDSC assessments

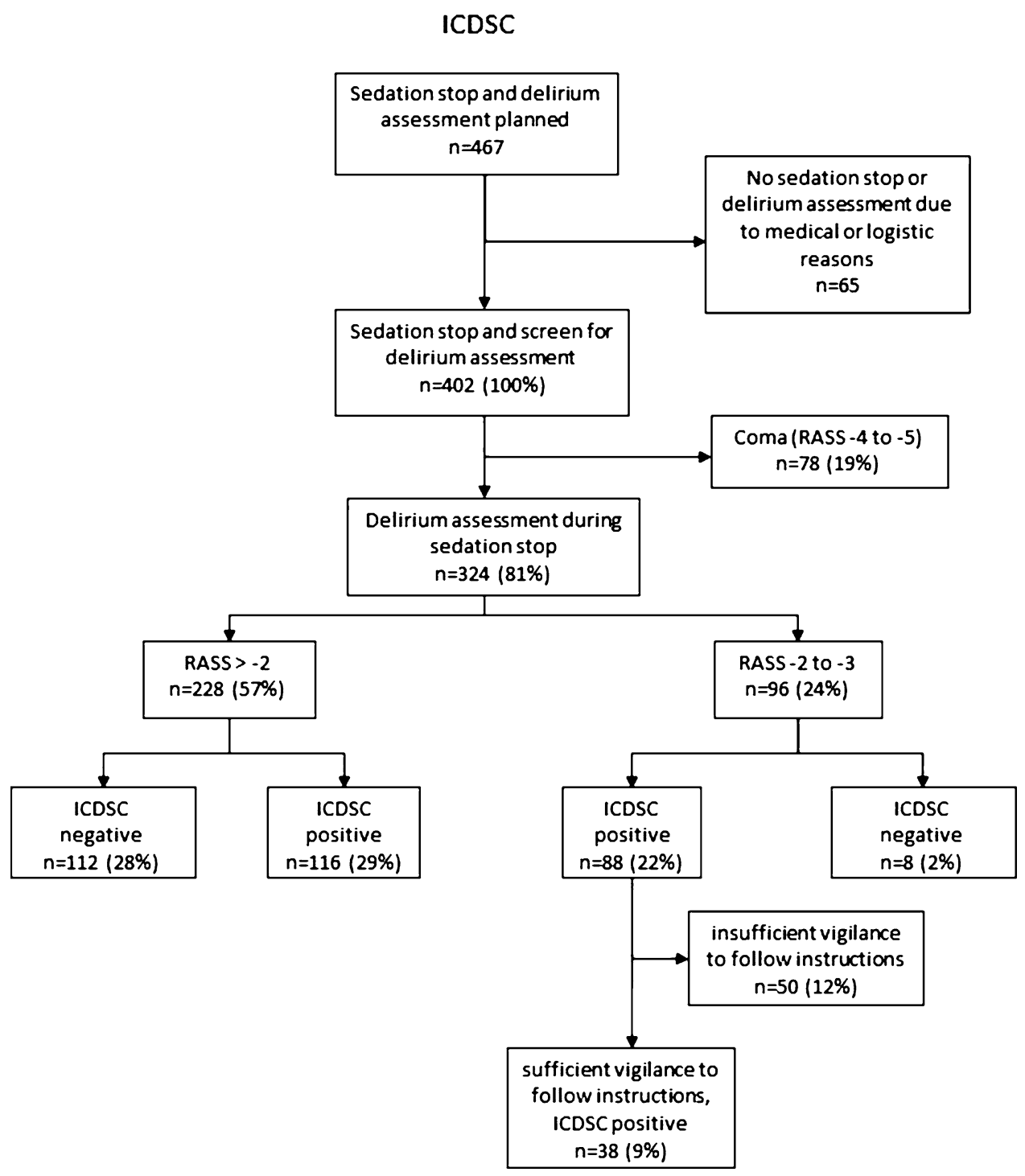

\section{Discussion}

We found that in $20 \%$ of all sedation stops, during delirium assessment, patients remained moderately sedated despite continuing sedation stops for up to $2 \mathrm{~h}$, or had their sedation restarted because of cardiovascular or respiratory instability, despite persisting moderate sedation. Furthermore, the apparent prevalence of delirium was dependent on how patients who remained at RASS 2 to -3 during sedation stops were classified into the three possible schemes we provided in the "Materials and methods" section. According to the CAM-ICU methodology, these patients formally qualify for delirium assessment. Our results demonstrate that possible persisting sedation during sedation stops may increase the apparent prevalence of delirium by approximately a quarter to a third when either CAM-ICU or ICDSC is used to diagnose delirium. The results were essentially similar if patients with primary neurologic diagnoses were excluded.

The proportion of assessments done during mechanical ventilation $(93 \%)$ was high and the prevalence of coma (11\% of patients, $19 \%$ of assessments) low in the present study as compared to previous studies [5, 7, 9]. The proportion of assessment done without sedation but intubated $(22 \%)$ was also high. These findings are consistent with our strategy to avoid deep sedation. The interaction of sedation with delirium assessment is likely to be even more relevant if deep sedation is used and if no sedation stops are implemented. The risk of sedation interfering with the diagnosis of delirium is inherent to both CAM-ICU and ICDSC, because the presence and impact of sedation was not taken into account in the validation of these methods. Sedation stops have been used in studies on ICU delirium relatively rarely (or were 
Fig. 3 Flowchart of patients with at least one positive CAMICU assessment. Asterisk these patients can either be always at this level or have assessments done at higher RASS levels with negative CAM-ICU

\section{CAM-ICU \\ incidence}

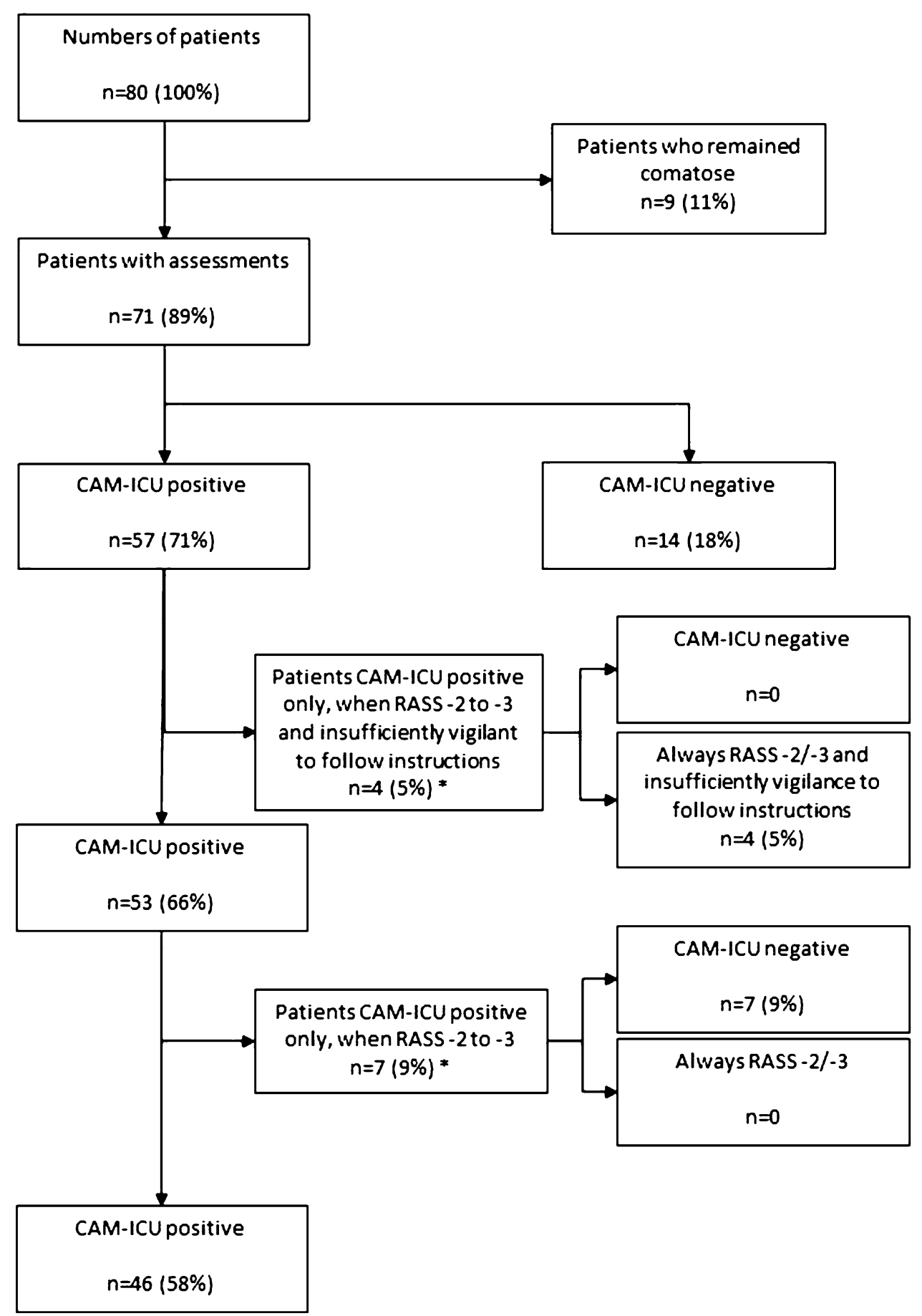

not reported). Since the effects of most commonly used sedative drugs can be prolonged, especially in long-term or high-dose use, sedation stops may help to reduce but not eliminate persisting effects of sedative drugs.
The CAM-ICU allows assessment at RASS -3 or higher (less sedated), either during ongoing sedation or sedation stops [17]. Most such patients will fulfill the criteria of acute onset of mental status change and 
Fig. 4 Flowchart of patients with at least one positive ICDSC assessment. Asterisk these patients can either be always at this level or have assessments done at higher RASS levels with negative ICDSC

\section{ICDSC \\ incidence}

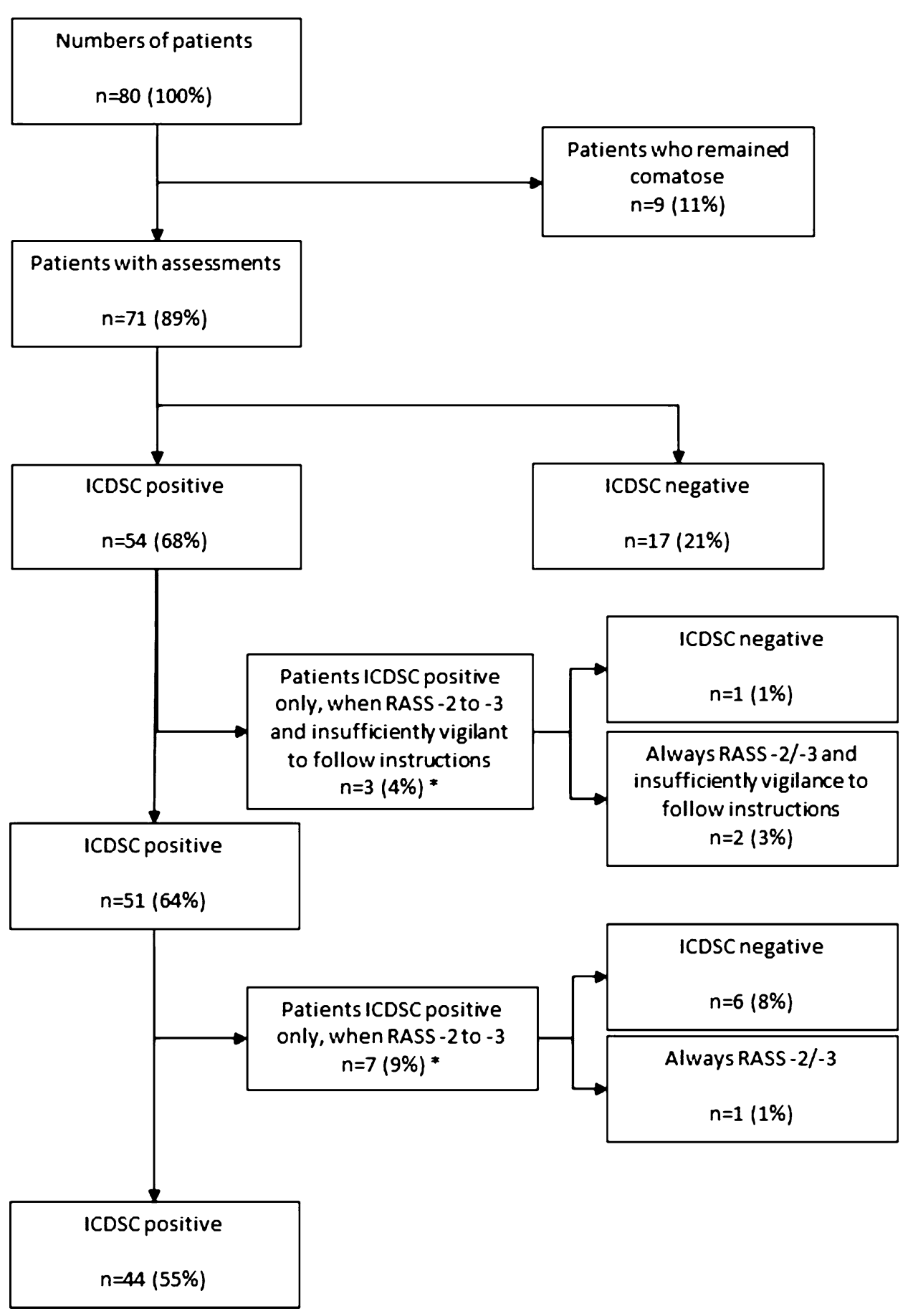

fluctuating course, in combination with altered level of consciousness; according to the CAM-ICU, this gives the diagnosis "delirium". Similarly, with the ICDSC, patients reacting to mild to moderate stimuli after discontinuing sedation qualify for assessment and are likely to demonstrate impaired levels of consciousness, inattention, psychomotor retardation, and fluctuation of symptoms, and thus to receive a diagnosis of "delirium". Recently, the new DSM-V criteria for delirium have been published [18]. The authors propose that noncomatose patients with 
reduced level of arousal of acute onset should be classified as having severe inattention and cognitive change, and hence delirium. Although we agree that such patients can be delirious, it should be noted that the same criteria-which correspond to a RASS -2/-3 level—can also apply for sedated healthy volunteers [19], and patients with uneventful postoperative course during awakening from anesthesia [20]. In patients who received sedative drugs prior to delirium assessment, it is therefore difficult or impossible to attribute these symptoms to either persistent sedation or delirium. In both cases, potential reasons for delirium should be eliminated, and sedatives kept stopped. There is no proven pharmacological treatment for hypoactive delirium. We suggest that any reports on delirium should be stratified for the RASS score during the assessment. Sedation stops should be added to delirium assessment and RASS level and reasons necessitating reinstitution of sedation reported.

Our study has limitations. The number of patients was relatively small. We did not use a reference "gold standard" assessment of delirium, such as one performed by a trained psychiatrist. High inter-rater agreement in routine delirium scoring after minimal training has been observed [15]. Recently, the sensitivity of CAM-ICU has been questioned, when CAM-ICU assessment by the bedside staff was compared with delirium assessments performed by delirium experts [9]. Our approach demonstrates results from a clinically relevant "real-world" environment rather than from dedicated specialist delirium assessors who are usually not present in the ICU. A further limitation is the assessment of delirium after a maximum of 120 min of sedation stop which was done for practical reasons. An interesting approach would have been to evaluate delirium before and after sedation stop and to include a multivariate analysis of the risk factors of patients diagnosed with delirium at a level of consciousness of RASS -2 to -3 .

The classification of patients remaining at level RASS -2 to -3 as "not assessable" only for those events where, according to the investigator, the patient was unable to maintain vigilance to understand the instructions of the letters attention test or the alternating pictures test of CAM-ICU is a subjective and non-validated approach. It should not be regarded as a new method of using the delirium assessment tools.

In conclusion, our study demonstrates that results from assessments using the current delirium screening tools depend on the level of sedation. In sedated patients, CAM-ICU and ICDSC may also measure pure sedation effects, and are therefore likely to overestimate the prevalence of delirium, especially hypoactive delirium. We suggest that reports on delirium using these scores should be stratified for the RASS score during the assessment and that sedation stops should be prolonged until no change in RASS occurs before assessing delirium.

Acknowledgments The authors would like to thank the physicians and the nursing staff and the study nurse team of the ICU for their invaluable support.

Conflicts of interest This study was supported entirely by departmental resources. None of the authors reports a conflict of interest.

\section{References}

1. Girard TD, Pandharipande PP, Ely EW (2008) Delirium in the intensive care unit. Crit Care 12(Suppl 3):S3. doi: 10.1186/cc6149

2. Pisani MA, Kong SY, Kasl SV, Murphy TE, Araujo KL, Van Ness PH (2009) Days of delirium are associated with 1-year mortality in an older intensive care unit population. Am J Respir Crit Care Med 180:1092-1097. doi: 10.1164/rccm.200904-0537OC

3. Ouimet S, Kavanagh BP, Gottfried SB, Skrobik Y (2007) Incidence, risk factors and consequences of ICU delirium. Intensive Care Med 33:66-73. doi: 10.1007/s00134-006-0399-8

4. Ely EW, Gautam S, Margolin R, Francis J, May L, Speroff T, Truman B, Dittus R, Bernard R, Inouye SK (2001) The impact of delirium in the intensive care unit on hospital length of stay. Intensive Care Med 27:1892-1900. doi: 10.1007/s00134-001-1132-2
5. Ely EW, Shintani A, Truman B, Speroff $\mathrm{T}$, Gordon SM, Harrell FE Jr, Inouye SK, Bernard GR, Dittus RS (2004) Delirium as a predictor of mortality in mechanically ventilated patients in the intensive care unit. JAMA 291:1753-1762

6. Ely EW, Margolin R, Francis J, May L, Truman B, Dittus R, Speroff T, Gautam S, Bernard GR, Inouye SK (2001) Evaluation of delirium in critically ill patients: validation of the Confusion Assessment Method for the Intensive Care Unit (CAM-ICU). Crit Care Med 29:1370-1379 (pii 11445689)

7. Ely EW, Inouye SK, Bernard GR, Gordon S, Francis J, May L, Truman B, Speroff T, Gautam S, Margolin R, Hart RP, Dittus R (2001) Delirium in mechanically ventilated patients: validity and reliability of the Confusion Assessment Method for the Intensive Care Unit (CAM-ICU). JAMA 286:2703-2710 (pii 11730446)
8. Bergeron N, Dubois MJ, Dumont M, Dial S, Skrobik Y (2001) Intensive Care Delirium Screening Checklist: evaluation of a new screening tool. Intensive Care Med 27:859-864 (pii 11430542)

9. van Eijk MM, van den Boogaard M, van Marum RJ, Benner P, Eikelenboom $\mathrm{P}$, Honing ML, van der Hoven B, Horn J, Izaks GJ, Kalf A, Karakus A, Klijn IA, Kuiper MA, de Leeuw FE, de Man T, van der Mast RC, Osse RJ, de Rooij SE, Spronk PE, van der Voort PH, van Gool WA, Slooter AJ (2011) Routine use of the Confusion Assessment Method for the Intensive Care Unit: a multicenter study. Am J Respir Crit Care Med 184:340-344 
10. Pandharipande P, Cotton BA, Shintani A, Thompson J, Costabile S, Truman Pun B, Dittus R, Ely EW (2007) Motoric subtypes of delirium in mechanically ventilated surgical and trauma intensive care unit patients. Intensive Care Med 33:1726-1731. doi: 10.1007/s00134-007-0687-y

11. Peterson JF, Pun BT, Dittus RS, Thomason JW, Jackson JC, Shintani AK, Ely EW (2006) Delirium and its motoric subtypes: a study of 614 critically ill patients. J Am Geriatr Soc 54:479-484. doi: 10.1111/j.1532-5415.2005.00621.x

12. Spronk PE, Riekerk B, Hofhuis J, Rommes JH (2009) Occurrence of delirium is severely underestimated in the ICU during daily care. Intensive Care Med 35:1276-1280

13. Ouimet S, Riker R, Bergeron N, Cossette M, Kavanagh B, Skrobik Y (2007) Subsyndromal delirium in the ICU: evidence for a disease spectrum. Intensive Care Med 33:1007-1013. doi: 10.1007/s00134-007-0618-y
14. Jakob SM, Lubszky S, Friolet R, Rothen HU, Kolarova A, Takala J (2007) Sedation and weaning from mechanical ventilation: effects of process optimization outside a clinical trial. J Crit Care 22:219-228. doi: 10.1016/j.jcrc.2007.01.001

15. Pun BT, Gordon SM, Peterson JF, Shintani AK, Jackson JC, Foss J, Harding SD, Bernard GR, Dittus RS, Ely EW (2005) Large-scale implementation of sedation and delirium monitoring in the intensive care unit: a report from two medical centers. Crit Care Med 33:1199-1205 (pii 15942331)

16. Soja SL, Pandharipande PP, Fleming SB, Cotton BA, Miller LR, Weaver SG, Lee BT, Ely EW (2008) Implementation, reliability testing, and compliance monitoring of the Confusion Assessment Method for the Intensive Care Unit in trauma patients. Intensive Care Med 34:1263-1268. doi: 10.1007/s00134-008-1031-x

17. Ely EW (2010) Confusion Assessment Method for the ICU (CAM-ICU) the complete training manual. http:// www.mc.vanderbilt.edu/icudelirium/ docs/CAM_ICU_training.pdf. Accessed 15 Mar 2013
18. Association AP (2013) Delirium diagnostic and statistical manual of mental disorders DSM-5. American Psychiatric Publishing, Arlington, pp 596-602

19. Haenggi M, Ypparila H, Takala J, Korhonen I, Luginbuhl M, PetersenFelix S, Jakob SM (2004) Measuring depth of sedation with auditory evoked potentials during controlled infusion of propofol and remifentanil in healthy volunteers. Anesth Analg 99:1728-1736. doi:10.1213/01. ANE.0000135634.46493.0A (table of contents)

20. Haenggi M, Ypparila-Wolters H, Buerki S, Schlauri R, Korhonen I, Takala J, Jakob SM (2009) Auditory event-related potentials, bispectral index, and entropy for the discrimination of different levels of sedation in intensive care unit patients. Anesth Analg 109:807-816. doi: 10.1213/ane.0b013e3181acc85d 